\title{
THE SPACE OF PETTIS INTEGRABLE FUNCTIONS IS BARRELLED
}

\author{
LECH DREWNOWSKI, MIGUEL FLORENCIO, AND PEDRO J. PAÚL
}

(Communicated by William J. Davis)

\begin{abstract}
It is well known that the normed space of Pettis integrable functions from a finite measure space to a Banach space is not complete in general. Here we prove that this space is always barrelled; this tells us that we may apply two important results to this space, namely, the Banach-Steinhaus uniform boundedness principle and the closed graph theorem. The proof is based on a theorem stating that a quasi-barrelled space having a convenient Boolean algebra of projections is barrelled. We also use this theorem to give similar results for the spaces of Bochner integrable functions.
\end{abstract}

\section{BARRELLEDNESS OF SPACES \\ WITH BOOLEAN ALGEBRAS OF PROJECTIONS}

This paper deals with some conditions, related to Boolean algebras of projections modelled over a finite measure space, under which quasi-barrelled spaces are indeed barrelled. Barrelled spaces were introduced as the "good" class of locally convex spaces, as domain spaces for operators, for which uniform boundedness principles and closed graph theorems hold (see [6, §39.5(1), §34.6(9)] or $[10,4.1 .3,4.1 .10,7.1 .12])$. Although we refer the reader to the monographs $[2,6,10]$ for the terms used in this paper, we want to recall briefly the most relevant definitions.

Let $E$ be a locally convex space with dual $E^{\prime}$. Denote, respectively, by $\sigma\left(E^{\prime}, E\right)$ and $\beta\left(E^{\prime}, E\right)$ the corresponding weak and strong topologies of the duality on $E^{\prime}$. A subset $T$ of $E$ is said to be a barrel if it is absolutely convex, closed, and absorbent. If a barrel $T$ absorbs every bounded subset of $E$ then it is called bornivorous. Barrels are the polars of $\beta\left(E^{\prime}, E\right)$-bounded subsets of $E^{\prime}$. A locally convex space $E$ is said to be barrelled (resp. quasibarrelled) if every barrel (resp. bornivorous barrel) is a zero-neighborhood. Equivalently, $E$ is barrelled (resp. quasi-barrelled) if and only if every subset of $E^{\prime}$ that is $\sigma\left(E^{\prime}, E\right)$-bounded, i.e., pointwise bounded on $E$ (resp. $\beta\left(E^{\prime}, E\right)$ bounded, i.e., uniformly bounded on the bounded subsets of $E$ ) is equicontinuous. Metrizable locally convex spaces are quasi-barrelled and Banach (and

Received by the editors July 6, 1990 and, in revised form, August 30, 1990.

1991 Mathematics Subject Classification. Primary 46A08, 46E40; Secondary 46G10.

Key words and phrases. Barrelled spaces, Pettis integral, Bochner integral, Boolean algebras of projections. 
Fréchet) spaces are barrelled; on the other hand, there are normed spaces that are not barrelled [10, 4.1.8].

In what follows, $(\Omega, \Sigma, \mu)$ stands for a measure space, where $\mu$ is a finite, positive, countably additive measure defined on a $\sigma$-algebra $\Sigma$ of subsets of $\Omega$.

We are going to use a variant, stated in the next definition, of the concept of Boolean algebra of projections introduced by W. G. Bade (see [13] for a good list of references about this topic).

Definition. We say that a locally convex space $E$ admits an $(\Omega, \Sigma, \mu)$-Boolean algebra of projections if there exists a set $\left\{P_{A}: A \in \Sigma\right\}$ of linear projections in $E$ such that:

(1) $P_{\Omega}$ is the identity on $E, P_{A \cap B}=P_{A} \cdot P_{B}$ for all $A, B \in \Sigma$, and $P_{A \cup B}=$ $P_{A}+P_{B}$ for all disjoint $A, B \in \Sigma$.

(2) $P_{A}$ is continuous for every $A \in \Sigma$.

(3) For every $x \in E$, the vector measure $F_{x}: \Sigma \rightarrow E$ defined by $F_{x}(A):=$ $P_{A}(x)$ is $\mu$-continuous, meaning $\lim _{\mu(A) \rightarrow 0} P_{A}(x)=0$. (Note that this condition implies that $F_{x}$ is countably additive $[2$, I.2.4] and, in particular, bounded.)

The standard examples of Boolean algebras of projections arise in the cases when $E$ is a space of measurable functions (or classes of functions) defined on $\Omega$ and the projections are defined by $P_{A}(x):=\chi_{A} \cdot x$, where $\chi_{A}$ is the characteristic function of $A \in \Sigma$. In these examples, conditions (1)-(3) are usually easy to verify or well known.

Our main result, Theorem 1 below, will be applied only to metrizable spaces in the next sections of this paper. However, we think that it is of independent interest and may be applied to more general situations; hence, we state and prove Theorem 1 for quasi-barrelled spaces.

We begin by recalling a useful fact about barrels that will be used in the sequel (see [10, 3.1.3]).

Fact. Let $E$ be a locally convex space with dual $E^{\prime}$. If a barrel $T$ in $E$ absorbs all null sequences in $E$ then $T$ is bornivorous. Dually, if $M$ is a $\sigma\left(E^{\prime}, E\right)$ bounded subset of $E^{\prime}$ and $M$ is uniformly bounded on every null sequence in $E$, then $M$ is $\beta\left(E^{\prime}, E\right)$-bounded.

Lemma 1. Let $E$ be a quasi-barrelled locally convex space admitting an $(\Omega, \Sigma, \mu)$-Boolean algebra of projections $\left\{P_{A}: A \in \Sigma\right\}$; then $\left\{P_{A}: A \in \Sigma\right\}$ is equicontinuous.

Proof. Let $U$ be an absolutely convex and closed zero-neighborhood in $E$. Consider the set $T:=\left\{x \in E: P_{A}(x) \in U\right.$ for every $\left.A \in \Sigma\right\}$. It is clear that $T$ is absolutely convex and closed. $T$ is also absorbent because for every $x \in E$ the range of the vector measure $F_{x}$ is bounded in $E$. Hence $T$ is a barrel. Now, let $\left(x_{n}\right)$ be a null sequence in $E$. Since every $P_{A}$ is continuous, we have

$$
\lim _{n} F_{x_{n}}(A)=\lim _{n} P_{A}\left(x_{n}\right)=0 \text { for all } A \in \Sigma \text {. }
$$

This implies that $\left\{F_{x_{n}}(A): n=1,2, \ldots\right\}$ is bounded for every $A \in \Sigma$, so that we may apply the Nikodým Boundedness Theorem [2, I.3.1] to deduce that $\left\{P_{A}: A \in \Sigma\right\}$ is uniformly bounded on $\left(x_{n}\right)$ and, therefore, that $T$ absorbs $\left(x_{n}\right)$. The fact quoted above tells us that $T$ is a bornivorous barrel so that, since 
$E$ is quasi-barrelled, $T$ is a zero-neighborhood. This proves the equicontinuity of $\left\{P_{A}: A \in \Sigma\right\}$. Q.E.D.

Theorem 1. Let $E$ be a quasi-barrelled locally convex space admitting an $(\Omega, \Sigma, \mu)$-Boolean algebra of projections $\left\{P_{A}: A \in \Sigma\right\}$ such that the following condition is satisfied:

(4) Whenever $\left(A_{n}\right)$ is a disjoint sequence in $\Sigma$ and $\left(x_{n}\right)$ is a null sequence in $E$ such that

$$
P_{A_{n}}\left(x_{n}\right)=x_{n}, \quad n=1,2, \ldots,
$$

then there exists $x \in E$ and a sequence $n(1)<n(2)<\cdots$ in $\mathbb{N}$ such that

$$
P_{A_{n(k)}}(x)=x_{n(k)}, \quad k=1,2, \ldots .
$$

(Equivalently, there exists a sequence $n(1)<n(2)<\cdots$ in $\mathbb{N}$ such that $\sum x_{n(k)}$ converges.)

If $(\Omega, \Sigma, \mu)$ is atomless, then $E$ is barrelled.

Proof. According to the Fact above, we have to prove that if $M \subset E^{\prime}$ is $\sigma\left(E^{\prime}, E\right)$-bounded and $\left(x_{m}\right)$ is a null sequence in $E$, then

$$
\sup \left\{\left|\left\langle u, x_{m}\right\rangle\right|: m \in \mathbb{N}, u \in M\right\}<+\infty .
$$

Suppose, on the contrary, that this supremum is $+\infty$. We start by making a sliding-hump type inductive construction.

Step 1. Call $\Omega_{0}:=\Omega$; on account of our assumption we have

$$
\sup \left\{\left|\left\langle u, P_{\Omega_{0}}\left(x_{m}\right)\right\rangle\right|: m \in \mathbb{N}, u \in M\right\}=+\infty,
$$

and, therefore, we can find $u_{1} \in M$ and $m(1) \in \mathbb{N}$ such that

$$
\gamma_{1}:=\left|\left\langle u_{1}, P_{\Omega_{0}}\left(x_{m(1)}\right)\right\rangle\right|>1 \text {. }
$$

Applying condition (3) to $x_{m(1)}$, there exists $\delta>0$ such that whenever $\mu(C) \leq$ $\delta$,

$$
\left|\left\langle u_{1}, P_{C}\left(x_{m(1)}\right)\right\rangle\right|<\gamma_{1}-1 .
$$

Since the measure space is atomless, $\Omega_{0}$ can be written as a finite union of measurable sets of measure at most $\delta$. By $(*)$, on one of these sets, say $\Omega_{1}$, the corresponding supremum must be infinite; hence

$$
\sup \left\{\left|\left\langle u, P_{\Omega_{1}}\left(x_{m}\right)\right\rangle\right|: m>m(1), u \in M\right\}=+\infty .
$$

Set $A_{1}:=\Omega_{0} \backslash \Omega_{1}$; then, because $\mu\left(\Omega_{1}\right) \leq \delta$, we have

$$
\begin{aligned}
\left|\left\langle u_{1}, P_{A_{1}}\left(x_{m(1)}\right)\right\rangle\right| & =\left|\left\langle u_{1}, P_{\Omega_{0}}\left(x_{m(1)}\right)\right\rangle-\left\langle u_{1}, P_{\Omega_{1}}\left(x_{m(1)}\right)\right\rangle\right| \\
& \geq\left|\left\langle u_{1}, P_{\Omega_{0}}\left(x_{m(1)}\right)\right\rangle\right|-\left|\left\langle u_{1}, P_{\Omega_{1}}\left(x_{m(1)}\right)\right\rangle\right| \\
& >\gamma_{1}-\left(\gamma_{1}-1\right)=1 .
\end{aligned}
$$

Step 2. We start from (**) finding $u_{2} \in M$ and $m(2)>m(1)$ such that

$$
\left|\left\langle u_{2}, P_{\Omega_{1}}\left(x_{m(2)}\right)\right\rangle\right|>2 .
$$

Proceeding as in Step 1, we can find a set $\Omega_{2} \subset \Omega_{1}$ such that

$$
\sup \left\{\left|\left\langle u, P_{\Omega_{2}}\left(x_{m}\right)\right\rangle\right|: m>m(2), u \in M\right\}=+\infty
$$

and $\left|\left\langle u_{2}, P_{A_{2}}\left(x_{m(2)}\right)\right\rangle\right|>2$, where $A_{2}:=\Omega_{1} \backslash \Omega_{2}$. 
In this way, we are able to find a sequence $\left(u_{n}\right)$ in $M$, a disjoint sequence $\left(A_{n}\right)$ in $\Sigma$, and a subsequence $\left(x_{m(n)}\right)$ of $\left(x_{n}\right)$ such that

$$
\left|\left\langle u_{n}, P_{A_{n}}\left(x_{m(n)}\right)\right\rangle\right|>n, \quad n=1,2, \ldots .
$$

Since $x_{m(n)} \rightarrow 0$ and, by the lemma above , $\left\{P_{A}: A \in \Sigma\right\}$ is equicontinuous, we have $\lim _{n} P_{A_{n}}\left(x_{m(n)}\right)=0$ so that we may apply condition (4): there exists $y \in E$ and a subsequence $n(1)<n(2)<\cdots$ such that

$$
P_{A_{n(k)}}(y)=P_{A_{n(k)}}\left(x_{m(n(k))}\right), \quad k=1,2, \ldots .
$$

For the sake of simplicity, let us write $v_{k}:=u_{n(k)}$ and $B_{k}:=A_{n(k)}$; then

$$
\left|\left\langle v_{k}, P_{B_{k}}(y)\right\rangle\right|>n(k)>k, \quad k=1,2, \ldots .
$$

Since $M$ is $\sigma\left(E^{\prime}, E\right)$-bounded, the scalar measures

$$
m_{k}: A \rightarrow m_{k}(A):=\left\langle v_{k}, P_{A}(y)\right\rangle, \quad k=1,2, \ldots
$$

form a setwise bounded set, i.e.,

$$
\sup \left\{\left|m_{k}(A)\right|: k=1,2, \ldots\right\}<+\infty \quad \text { for all } A \in \Sigma .
$$

Therefore, we may apply the Nikodým Boundedness Theorem [2, I.3.1] to deduce that

$$
\sup \left\{\left|m_{k}(A)\right|: A \in \Sigma, k=1,2, \ldots\right\}<+\infty,
$$

in contradiction with the fact that

$$
\left|m_{k}\left(B_{k}\right)\right|=\left|\left\langle v_{k}, P_{B_{k}}(y)\right\rangle\right|>k, \quad k=1,2, \ldots \text { Q.E.D. }
$$

Corollary. Let $E$ be a metrizable locally convex space admitting an $(\Omega, \Sigma, \mu)$ Boolean algebra of projections $\left\{P_{A}: A \in \Sigma\right\}$ and satisfying condition (4) of Theorem 1. If $F$ is a closed subspace of $E$ such that $P_{A}(F) \subset F$ for all $A \in \Sigma$ and $(\Omega, \Sigma, \mu)$ is atomless, then $F$ is barrelled.

Remark. A locally convex space $E$ is said to have property $(\mathrm{K})$ if every null sequence $\left(x_{n}\right)$ in $E$ has a subsequence $\left(x_{n(k)}\right)_{k}$ such that $\sum x_{n(k)}$ is convergent. This notion was introduced by S. Mazur and W. Orlicz and rediscovered by P. Antosik. Property (K) has been used to give noncategorical proofs of several classical theorems, as well as some new results, about open mappings, closed graphs, and barrelledness (see [1] and references therein or [10, 1.2.15, 1.4]).

It is clear that property $(\mathrm{K})$ implies condition (4) for spaces admitting an $(\Omega, \Sigma, \mu)$-Boolean algebra of projections. On the other hand, we shall give in this paper two examples, in Remark 3 of $\S 2$ and Remark 2 of $\S 3$, of spaces satisfying condition (4) but not property $(\mathrm{K})$. Note, however, that the theorem of [1] states that every metrizable space having property $(\mathrm{K})$ is barrelled, not needing the hypothesis of the existence of an $(\Omega, \Sigma, \mu)$-Boolean algebra of projections.

\section{Application to spaces of Pettis integrable functions}

Let $X$ be a Banach space. A weakly $\mu$-measurable function $f: \Omega \rightarrow X$ is said to be Pettis integrable if the composition $t \rightarrow\left\langle x^{*}, f(t)\right\rangle$ is a function in $L_{1}(\mu)$ for every $x^{*} \in X^{\prime}$ and if for every measurable set $A$ there is an element in $X$ called the Pettis integral of $f$ over $A$ and denoted by $\int_{A} f d \mu$ such that

$$
\left\langle x^{*}, \int_{A} f d \mu\right\rangle=\int_{A}\left\langle x^{*}, f(t)\right\rangle d \mu(t) \text { for all } x^{*} \in X^{\prime} .
$$


We shall denote by $\mathscr{P}(\mu, X)$ the space of (classes of) Pettis integrable functions $f: \Omega \rightarrow X$, endowed with its natural norm given by the formula

$$
\|f\|:=\sup \left\{\int_{\Omega}\left|\left\langle x^{*}, f(t)\right\rangle\right| d \mu(t): x^{*} \in X^{\prime},\left\|x^{*}\right\| \leq 1\right\} .
$$

Note that $\left\|\int_{A} f d \mu\right\| \leq\|f\|$ for every $f \in \mathscr{P}(\mu, X)$ and $A \in \Sigma$. For more details, we refer the reader to $[2$, II.3; $4,3.7]$ and the original paper by Pettis $[11, \S 3]$.

For the case when $\mu$ is the Lebesgue measure in $[0,1]$, Pettis showed that the normed space $\mathscr{P}\left(\mu, L_{2}(\mu)\right)$ is noncomplete [11,9.4]. Later, it was proved by Thomas [14, p. 131] and Janicka and Kalton [5] that the same holds if we replace $L_{2}$ with an arbitrary infinite-dimensional Banach space; the former author remarks that the result is valid for any atomless finite measure space.

We shall prove that $\mathscr{P}(\mu, X)$ is barrelled for every finite measure space. As we pointed out in the previous section, this result will make it possible to apply the Banach-Steinhaus and Closed Graph Theorems to $\mathscr{P}(\mu, X)$, in spite of the fact that this space is noncomplete, in general.

Theorem 2. Let $X$ be any Banach space; then the normed space $\mathscr{P}(\mu, X)$ of Pettis integrable functions is barrelled.

Proof. Assume first that $(\Omega, \Sigma, \mu)$ is atomless. We shall apply Theorem 1 . For $A \in \Sigma$ and $f \in \mathscr{P}(\mu, X)$, define $P_{A}(f):=\chi_{A} \cdot f$. It is clear that $P_{A}$ is a linear projection in $\mathscr{P}(\mu, X)$ and, since $\left\|P_{A}(f)\right\|=\left\|\chi_{A} \cdot f\right\| \leq\|f\|$, it follows that $\left\{P_{A}: A \in \Sigma\right\}$ is equicontinuous.

Condition (3) holds by [11, 2.51] (see also [4, 3.7.2]).

Condition (4): Let $\left(A_{n}\right)$ be a disjoint sequence in $\Sigma$ and $\left(f_{n}\right)$ a null sequence in $\mathscr{P}(\mu, X)$ with $\chi_{A_{n}} \cdot f_{n}=f_{n}$. We show that any absolutely summable subsequence of $\left(f_{n}\right)$ fulfills the requirements in (4). Without loss of generality, assume that $\alpha:=\sum_{n}\left\|f_{n}\right\|$ is finite. Since the $f_{n}$ are disjointly supported, the pointwise sum $f(t):=\sum_{n} f_{n}(t)$ exists for all $t \in \Omega ; \chi_{A_{n}} \cdot f=f_{n}$ for every $n=1,2, \ldots$; and for every $x^{*} \in X^{\prime}$ we have

$$
\int_{\Omega}\left|\left\langle x^{*}, f(t)\right\rangle\right| d \mu(t)=\int_{\Omega} \sum_{n=1}^{\infty}\left|\left\langle x^{*}, f_{n}(t)\right\rangle\right| d \mu(t) \leq \alpha\left\|x^{*}\right\|,
$$

so that $\left\langle x^{*}, f(\cdot)\right\rangle$ is in $L_{1}(\mu)$. Moreover, for every $A \in \Sigma$, we have $\sum_{n}\left\|\int_{A} f_{n} d \mu\right\| \leq \alpha$, hence the series $\sum_{n} \int_{A} f_{n} d \mu$ of the corresponding Pettis integrals converges to some element $x_{A}$ in the Banach space $X$. Now, for every $x^{*} \in X^{\prime}$ and $A \in \Sigma$ we have

$$
\left\langle x^{*}, x_{A}\right\rangle=\sum_{n=1}^{\infty}\left\langle x^{*}, \int_{A} f_{n} d \mu\right\rangle=\sum_{n=1}^{\infty} \int_{A \cap A_{n}}\left\langle x^{*}, f(t)\right\rangle d \mu(t)=\int_{A}\left\langle x^{*}, f(t)\right\rangle d \mu(t) .
$$

Hence $f$ is Pettis integrable. This finishes the proof for the case when $(\Omega, \Sigma, \mu)$ is atomless.

Now suppose that $(\Omega, \Sigma, \mu)$ contains atoms. Write $\Omega$ as $\Omega_{n A} \cup \Omega_{A}$, where $\mu$ is atomless on $\Omega_{n A}$ and purely atomic on $\Omega_{A}$. It is clear that $\mathscr{P}(\mu, X)$ is the topological direct sum of the spaces of Pettis integrable functions on $\Omega_{n A}$ and $\Omega_{A}$, respectively. Of these two spaces, the one corresponding to $\Omega_{n A}$ is barrelled as we have seen. On the other hand, if there is a finite number $m$ 
of atoms, the space corresponding to $\Omega_{A}$ is isomorphic to $X^{m}$; and, on the contrary, if the number of atoms is countable then it can be identified with the Banach space of unconditionally convergent series in $X$. Thus in any case, $\mathscr{P}(\mu, X)$ is the topological direct sum of a barrelled space plus a Banach space, hence it is barrelled. Q.E.D.

Remarks. 1. The proof of Theorem 2 can be altered to study the subspace $\mathscr{P}_{q}(\mu, X)$ of $\mathscr{P}(\mu, X)$ formed by those functions $f: \Omega \rightarrow X$ with $\left\langle x^{*}, f(\cdot)\right\rangle$ in $L_{q}(\mu)$ for every $x^{*} \in X^{\prime}$ [11]; when endowed with the norm

$$
\|f\|_{q}:=\sup \left\{\left\|\left\langle x^{*}, f(\cdot)\right\rangle\right\|_{q}: x^{*} \in X^{\prime},\left\|x^{*}\right\| \leq 1\right\},
$$

$\mathscr{P}_{q}(\mu, X)$ is a noncomplete (in general) normed space. It can be proved, using the same technique as above, that this space is barrelled as well.

2. The space $\mathscr{P}(\mu, X)$ is also barrelled when the measure space is $\sigma$-finite. The proof that we have found of this result, however, does not follow directly from Theorem 2; it is a consequence of a generalization of the sliding-hump technique of Theorem 1. This, and related results, will be included in a forthcoming paper [3], where we refer the interested reader.

3. We shall prove now that $\mathscr{P}\left(\mu, L_{2}(\mu)\right.$ ) (where $\mu$ is the Lebesgue measure on the unit interval) does not have property $(K)$. We use Pettis's example $[11,9.4]$ with a slight change of notation. Let $\left(\psi_{j}\right)$ be a complete orthonormal sequence in $L_{2}(\mu),\left(\Delta_{i}\right)$ a sequence of consecutive segments in $\mathbb{N}$ such that $\Delta_{i}$ has $2^{i}$ elements, and $\left(E_{j}\right)$ a sequence of intervals such that for $i \in \mathbb{N}$, the family $\left(E_{j}: j \in \Delta_{i}\right)$ is the partition of $[0,1)$ into subintervals $\left[(l-1) / 2^{i}, l / 2^{i}\right), l=1,2, \ldots, 2^{i}$. Consider the simple functions $f_{i}:[0,1] \rightarrow$ $L_{2}(\mu)$ defined by

$$
f_{i}(t):=\sum_{j \in \Delta_{i}} \psi_{j} \cdot \chi_{j}(t)
$$

where $\chi_{j}$ denotes the characteristic function of $E_{j}$. The sequence $\left(f_{i}\right)$ converges to zero in $\mathscr{P}\left(\mu, L_{2}(\mu)\right)$ [11, 9.4]. Suppose that a subseries $\sum_{k} f_{i(k)}$ converges in $\mathscr{P}\left(\mu, L_{2}(\mu)\right)$ to a function $f$. Then, for each fixed index $k_{0}$ and every $j \in \Delta_{i\left(k_{0}\right)}$, we have

$0=\lim _{n \rightarrow \infty} \int_{[0,1]}\left|\left\langle\psi_{j}, \sum_{k=1}^{n} f_{i(k)}(t)-f(t)\right\rangle\right| d \mu(t)=\int_{[0,1]}\left|\chi_{j}(t)-\left\langle\psi_{j}, f(t)\right\rangle\right| d \mu(t)$,

because, by orthonormality, $\sum_{k=1}^{n}\left\langle\psi_{j}, f_{i(k)}(t)\right\rangle=\chi_{j}(t)$ for $n \geq k_{0}$ and $t \in$ $[0,1]$. It follows that there is a $\mu$-null set $N_{j} \subset E_{j}$ such that $\left\langle\psi_{j}, f(t)\right\rangle=1$ for all $t \in E_{j} \backslash N_{j}$. Let $N$ be the union of all $N_{j}\left(j \in \Delta_{i(k)}, k=1,2, \ldots\right)$. Now, if $t \in[0,1]$, then for every $k \in \mathbb{N}$ there exists $j(k) \in \Delta_{i(k)}$ such that $t \in E_{j(k)}$. Hence, if additionally $t \notin N$, then $\left\langle\psi_{j(k)}, f(t)\right\rangle=1$ for every $k \in \mathbb{N}$. In consequence, for almost all $t \in[0,1], f(t)$ is a function in $L_{2}$ (remember $f:[0,1] \rightarrow L_{2}$ ) that has an infinite number of Fourier coefficients equal to 1 , which is clearly impossible.

\section{APPLICATION TO SPACES OF BOCHNER INTEGRABLE FUNCTIONS}

Let $L(q)$ be a solid Banach lattice of measurable scalar functions $\phi: \Omega \rightarrow \mathbb{R}$. (As usual, we identify functions that are equal $\mu$-a.e.) Thus if $\phi \in L$ and $\psi$ is a measurable function such that $|\psi(\cdot)| \leq|\phi(\cdot)| \mu$-a.e., then $\psi \in L$ and 
$q(\psi) \leq q(\phi)$. Examples of $L$ are the classical $L_{p}(\mu)$ spaces $(1 \leq p \leq \infty)$, Orlicz spaces, and Köthe normed spaces. These and more examples can be found in, e.g., [7, 9, 15], or [16, Chapter 15].

Recall that $L$ is said to be order-continuous if

$$
\lim _{\mu(A) \rightarrow 0} \chi_{A} \cdot \phi=0 \quad \text { for all } \phi \in L .
$$

This is satisfied, for instance, when $L=L_{p}(\mu) \quad(1 \leq p<\infty)$ or $L=L_{\Phi}(\mu)$ is an Orlicz space, where $\Phi$ satisfies the $\Delta_{2}$ condition.

Definition. Let $X$ be a normed space (not necessarily complete). We define the space $L(X)$ as the space of (classes of) strongly measurable functions $f: \Omega \rightarrow$ $X$ such that the scalar function $\phi(\cdot):=\|f(\cdot)\|$ is in $L$. We shall consider on $L(X)$ the topology defined by the norm $f \rightarrow q(\|f(\cdot)\|)$. In general, $L(X)$ will be noncomplete.

When $L=L_{p}(\mu)$ and $X$ is a dense subspace of a Banach space $Y, L(X)=$ $L_{p}(\mu, X)$ is a dense subspace of the space $L_{p}(\mu, Y)$ of $Y$-valued, Bochner $p$-integrable functions [2, II.2]. Other examples can be found in [16, Ch. 15].

Theorem 3. Let $L$ be an order-continuous solid Banach lattice of measurable functions defined over an atomless, finite measure space $(\Omega, \Sigma, \mu)$, and let $X$ be a normed space. Then the space $L(X)$, endowed with the corresponding normed topology, is barrelled. In particular, $L_{p}(X)$ is barrelled for $1 \leq p<\infty$. Proof. We shall apply Theorem 1. For $A \in \Sigma$ and $f \in L(X)$, define $P_{A}(f):=$ $\chi_{A} \cdot f$. Then it is clear, by the assumptions on $L$ and the definition of the topology in $L(X)$, that $\left\{P_{A}: A \in \Sigma\right\}$ is an $(\Omega, \Sigma, \mu)$-Boolean algebra of projections in $L(X)$.

To verify condition (4), let $\left(A_{n}\right)$ be a disjoint sequence in $\Sigma$ and $\left(f_{n}\right)$ a null sequence in $L(X)$ such that $P_{A_{n}}\left(f_{n}\right)=f_{n}$. The fact that $f_{n} \rightarrow 0$ in $L(X)$ implies that $\left\|f_{n}(\cdot)\right\| \rightarrow 0$ as functions in L. Since $L$ is a Banach space, there exists a subsequence $n(1)<n(2)<\cdots$ such that the series $\sum\left\|f_{n(k)}(\cdot)\right\|$ converges in $L$, and it is clear that the sum of this series is, precisely, the pointwise sum (in $\mathbb{R}$ ). Now, the function $f(\cdot):=\sum f_{n(k)}(\cdot)$ (pointwise sum in $X$ ) is strongly measurable and $\|f(\cdot)\|=\sum\left\|f_{n(k)}(\cdot)\right\| \in L$, so that $f \in L(X)$ and $f \cdot \chi_{A_{n(k)}}=f_{n(k)}$ for all $k=1,2, \ldots$ Q.E.D.

Remarks. 1. This theorem is a bit striking since we do not require $X$ to be barrelled. It is indeed the case that every separable Banach space $Y$ contains a dense subspace $X$ that is not barrelled and, nevertheless, we have that $L_{1}(\mu, X)$ is a dense barrelled subspace of $L_{1}(\mu, Y)$ if $(\Omega, \Sigma, \mu)$ is atomless.

2. Take $(\Omega, \Sigma, \mu)$ as the unit interval with Lebesgue measure and $X$ a normed space not satisfying property $(\mathrm{K})[10,1.2 .16(\mathrm{~b})]$. Then $L_{1}(X)$ satisfies condition (4) as we saw in the proof of Theorem 3, but $L_{1}(X)$ does not have property $(\mathbf{K})$ : simply note that the constant functions form a closed subspace of $L_{1}(X)$.

3. It is easy to see, using the same method of proof, that Theorem 3 can be extended to the case when $L$ is an order-continuous Fréchet lattice (some examples may be found in $[8,9,12])$. Moreover, using a diagonal procedure in the verification of condition (4), one can show that Theorem 3 also holds when $X$ is merely metrizable $(L(X)$ being defined in an analogous way). 
Note. M. Florencio, P. J. Paúl and C. Sáez gave a proof of Theorem 3 for the case of the space $L_{1}(X)$ under restrictive hypotheses, namely, that $(\Omega, \Sigma, \mu)$ is an atomless Radon measure space and $X^{\prime}$ satisfies the Radon-Nikodým property. This was presented to the II Conference on Function Spaces held September 1989 in Poznań, where the collaboration with L. Drewnowski started. The authors would like to thank F. Bombal (Madrid), F. Freniche (Sevilla), P. Greim (Charleston, South Carolina), and the referee for their helpful comments and remarks. Thanks also to La Consejería de Educación y Ciencia de la Junta de Andalucía for partially supporting the stays of P. J. Paúl at Poznań and L. Drewnowski at Seville.

\section{REFERENCES}

1. P. Antosik and J. Burzyk, Sequential conditions for barrelledness and bornology, Bull. Acad. Polon. Sci. Sér. Sci. Math. 35 (1987), 457-459.

2. J. Diestel and J. J. Uhl, Vector measures, Math Surveys and Mónographs, vol. 15, Amer. Math. Soc., Providence, RI, 1977.

3. L. Drewnowski, M. Florencio, and P. J. Paúl, Uniform boundedness of operators and barrelledness in spaces with Boolean algebras of projections. IV Convegno di Analisi Reale e Teoria de Misura, Capri, Italy, 1990, (to appear).

4. E. Hille and R. S. Phillips, Functional analysis and semi-groups, Colloq. Publ., vol. 31, Amer. Math. Soc., Providence, RI, 1957.

5. L. Janicka and N. J. Kalton, Vector measures of infinite variation, Bull. Acad. Polon. Sci. Sér. Sci. Math. Astronom. Phys. 25 (1977), 239-241.

6. G. Köthe, Topological vector spaces, I, II, Springer-Verlag, Berlin, Heidelberg, and New York, 1969, 1979.

7. J. Lindenstrauss and L. Tzafriri, Classical Banach spaces, II, Springer-Verlag, Berlin, Heidelberg, and New York, 1979.

8. J. A. López Molina, The dual and bidual of an echelon Köthe space, Collect. Math. 31 (1980), 159-191.

9. G. G. Lorentz and D. G. Wertheim, Representation of linear functionals on Köthe spaces, Canad. J. Math 5 (19j3), 568-575.

10. P. Pérez Carreras and J. Bonet, Barrelled locally convex spaces, North-Holland Mathematics Studies, vol. 131 North-Holland, Amsterdam, New York, Oxford, and Tokyo, 1987.

11. B. J. Pettis, On integiation in vector spaces, Trans. Amer. Math. Soc. 44 (1938), 277-304.

12. K. Reiher, Weigthed inductive and projective limits of normed Köthe function spaces, Resultate Math. 13 (1988), 147-161.

13. W. J. Ricker and $\mathrm{H}$. H. Schaefer, The uniformly closed algebra generated by a complete Boolean algebra of projections, Math. Z. 201 (1989), 429-439.

14. G. E. F. Thomas, Totally summable functions with values in locally convex spaces, Measure Theory (Proc. Conf. Oberwolfach, 1975), Lecture Notes in Math., vol. 541, Springer-Verlag, Berlin, Heidelberg, and New York, 1976, pp. 117-131.

15. R. Welland, On Köthe spaces, Trans. Amer. Math. Soc. 112 (1964), 267-277.

16. A. C. Zaanen, Integration, North-Holland, Amsterdam, 1967.

L. Drewnowski, Instytut Matematyki, Uniw. Adama Mickiewicza, ul. Matejki 48/49, 60-769 Poznań, Poland

M. Florencio, P. J. Paúl, E. S. Ingenieros Industriales, Avda. Reina Mercedes s/N, 41012 Sevilla, Spain 